\title{
ON SOME NONLINEAR INTEGRAL AND INTEGRO-DIFFERENTIAL EQUATIONS WITH NONCOMPACT OPERATORS ON POSITIVE HALF-LINE
}

\author{
M.F. BROYAN, KH.A. KHACHATRYAN
}

\begin{abstract}
The paper is devoted to the studying certain classes of nonlinear integral and integro-differential with non-compact Hammerstein type operators. These equations have important applications in the kinetic theory of gases and in the wealth distribution theory of a one product economics.
\end{abstract}

Keywords:integral equation, Hammerstein operator, Sobolev space, convergence, monotonicity.

\section{INTRODUCTION}

The work is devoted to the solvability in certain functional spaces of the following classes of nonlinear integral and integro-differential equations with a non-compact Hammerstein-WienerHopf type operator,

$$
\begin{gathered}
f(x)=\int_{0}^{\infty} K_{0}(x-t) N_{0}(t, f(t)) d t+\int_{0}^{\infty} K_{1}(x+t) N_{1}(t, f(t)) d t, \quad x>0, \\
\left\{\begin{array}{l}
\frac{d \varphi}{d x}+\lambda \varphi(x)=\int_{0}^{\infty} T(x-t) H(t, \varphi(t)) d t+\int_{0}^{\infty} T_{1}(x+t) H_{1}(t, \varphi(t)) d t, \quad x>0, \\
\varphi(0)=0
\end{array}\right.
\end{gathered}
$$

w.r.t. the functions $f(x)$ and $\varphi(x)$, respectively.

Apart from a mathematical interest, these classes of equations have direct applications in the kinetic theory of gases (equation (10) and in the econometric theory (problem (2)-(3)) (see [1]-44).

For equation (1) we suppose

$$
\begin{gathered}
K_{0}(x) \geq 0, \quad x \in \mathbb{R}, \quad K_{0} \in L_{1}(\mathbb{R}) \cap L_{\infty}(\mathbb{R}), \quad \int_{-\infty}^{+\infty} K_{0}(x) d x=1, \\
K_{1}(x) \geq 0, \quad K_{1} \neq \equiv, \quad \int_{x}^{\infty} K_{1}(\tau) d \tau \leqslant \int_{x}^{\infty} K_{0}(\tau) d \tau, \quad x \in \mathbb{R}^{+} \equiv(0,+\infty) .
\end{gathered}
$$

M.F. Broyan, Kh.A. Khachatryan, On some nonlinear integral and integro-differential EQUATIONS WITH NONCOMPACT OPERATORS ON POSITIVE SEMI AXIS.

(c) Broyan M.F., Khachatryan Kh.A. 2013.

Submitted January 25, 2012. 
In problem (2)-(3), $\lambda$ is a positive scalar parameter of equation (2), and kernels $T$ and $T_{1}$ satisfy the following conditions,

$$
\begin{gathered}
T_{1}(x) \geq 0, \quad T_{1} \not \equiv 0, \quad x \in \mathbb{R}^{+}, \quad T_{1} \in L_{1}\left(\mathbb{R}^{+}\right), \\
T(x) \geq 0, \quad x \in \mathbb{R}, \quad T \in L_{1}(\mathbb{R}), \quad \int_{-\infty}^{+\infty} T(x) d x=\lambda, \\
\int_{x}^{\infty} T_{1}(z) d z \leqslant \int_{x}^{\infty} T(z) d z, \quad x \in \mathbb{R}^{+}, \\
\nu(T) \equiv \int_{-\infty}^{+\infty} \tau T(\tau) d \tau<-1, \quad \int_{-\infty}^{+\infty}|\tau|^{j} T(\tau) d \tau<+\infty, \quad j=1,2 .
\end{gathered}
$$

$N_{0}, N_{1}, H$, and $H_{1}$ are real functions defined on the set $\mathbb{R}^{+} \times \mathbb{R}$ and satisfying certain conditions (see Theorems 1-3).

In the linear case, as $N_{0}(t, z) \equiv N_{1}(t, z) \equiv z$, numerous papers were devoted to studying equation (1) (see [5]-[8] and the references therein).

In the case $K_{0}(x)=K_{1}(x)=\frac{1}{\sqrt{\pi}} e^{-x^{2}}$ and $N_{0}(t, z)=N_{1}(t, z)=z^{p}, p \in(0,1)$, due to an important application in the $p$-adic string theory, equation (1) was studied in works [9]-[12].

In the case $N_{0}(t, z) \equiv G(z), N_{1}(t, z) \equiv G_{1}(z), \forall t \in \mathbb{R}^{+}$, where $G, G_{1} \in C[0, \eta], G(z) \geq z$, $G_{1}(z) \geq 0, z \in[0, \eta], G, G_{1} \uparrow$ on $[0, \eta]$ and $G(\eta)=G_{1}(\eta)=\eta$ for some $\eta>0$, equation (1) was studied in work [13] and the existence of a positive and bounded solution tending to $\eta$ at infinity was proven.

In the case $N_{0}(t, z) \equiv z-\omega(z), N_{1}(t, z) \equiv 0$, and $K_{0}(-x)=K_{0}(x), x>0, \int_{-\infty}^{+\infty}|x|^{j} K_{0}(x) d x<$ $+\infty, j=1,2$, where $0 \leqslant \omega \downarrow$ w.r.t. $z$ on $[A,+\infty), A>0, \omega \in C[A,+\infty) \cap L_{1}(0,+\infty)$, in work [14, the existence of a one-parametric family of positive solutions with the asymptotic behavior $O(x)$ as $x \rightarrow+\infty$ was proven. Later, in works [15, 16], this result was generalized first for the case $\nu\left(K_{0}\right) \leqslant 0, N_{0}(t, z) \equiv \mu(t)(z-\stackrel{\circ}{\omega}(t, z)), N_{1}(t, z) \equiv z$, where $0<\mu(t) \leqslant 1, t \in \mathbb{R}^{+}$, $1-\mu \in L_{1}\left(\mathbb{R}^{+}\right), \stackrel{\circ}{\omega}(t, z) \geq 0, \stackrel{\circ}{\omega}(t, z) \leqslant \omega(z),(t, z) \in \mathbb{R}^{+} \times[A,+\infty), \stackrel{\circ}{\omega} \downarrow$ w.r.t. $z$ on $[A,+\infty)$, and after that, in [17, 18, for the cases $N_{0}(t, z) \equiv \mu(t)(G(z)-\stackrel{\circ}{\omega}(t, z)), N_{1}(t, z) \equiv G_{1}(z)$.

Recently, in [19], problem (2)-(3) was studied in the case $H(t, z)=G(z), H_{1} \equiv 0$. In [19], a nonnegative and monotonically growing nonzero solution in the Sobolev space $W_{\infty}^{1}\left(\mathbb{R}^{+}\right)$was constructed.

In the present work we construct nonzero and nonnegative solutions to equations (1) and (2) for completely different conditions for $N_{0}, N_{1}, H$, and $H_{1}$. We note also that for various values of $\nu\left(K_{0}\right)$, a solution to equation (1) is constructed in the spaces $L_{1}\left(\mathbb{R}^{+}\right) \cap L_{\infty}^{0}\left(\mathbb{R}^{+}\right)$and $L_{\infty}^{0}\left(\mathbb{R}^{+}\right) \equiv\left\{\varphi(x): \varphi \in L_{\infty}\left(\mathbb{R}^{+}\right), \lim _{x \rightarrow \infty} \varphi(x)=0\right\}$, and under conditions (6)-(9), a solution to problem (2)-(3) is constructed in the Sobolev space $W_{1}^{1}\left(\mathbb{R}^{+}\right)$. 


\section{Solvability of EQUATION (1) In CASE OF NEGATIVIty OF FIRST MOMENT For KERNEL $K_{0}$}

Suppose for the functions $N_{0}(t, z)$ and $N_{1}(t, z)$ there exist numbers $\eta>0$ and $\eta_{0} \in(0, \eta)$ such that

1) $N_{0}(t, z), N_{1}(t, z) \uparrow$ w.r.t. $z$ on $\left[\Phi_{\eta_{0}}(t), \eta\right]$, for each fixed $t \in \mathbb{R}^{+}$, where

$$
\Phi_{\eta_{0}}(t) \equiv \eta_{0} \int_{t}^{\infty} K_{1}(\tau) d \tau, \quad t \in \mathbb{R}^{+} .
$$

2) $N_{0}$ and $N_{1}$ satisfy Caratheodory condition on the set $\mathbb{R}^{+} \times[0, \eta]$ w.r.t. $z$. In what follows, we write briefly this condition as

$$
\begin{array}{ll} 
& N_{0}, N_{1} \in \operatorname{Carat}_{z}\left(\mathbb{R}^{+} \times[0, \eta]\right), \\
\text { 3) } & N_{0}(t, 0) \equiv 0, \quad N_{1}(t, 0) \equiv 0, \quad t \in \mathbb{R}^{+} \\
\text {4) } & 0 \leqslant N_{0}(t, z) \leqslant z, \quad(t, z) \in \mathbb{R}^{+} \times\left[\Phi_{\eta_{0}}(t), \eta\right] \\
\text { 5) } & N_{1}\left(t, \Phi_{\eta_{0}}(t)\right) \geq \eta_{0}, \quad N_{1}(t, \eta) \leqslant \eta .
\end{array}
$$

The following theorem holds true.

Theorem 1. Suppose kernels $K_{0}$ and $K_{1}$ satisfy conditions (4)-(5) and $\nu\left(K_{0}\right) \equiv$ $\int_{-\infty}^{+\infty} \tau K_{0}(\tau) d \tau<0, \int_{-\infty}^{+\infty}|\tau|^{j} K_{0}(\tau) d \tau<+\infty, j=1,2$. Then equation (1) has a positive solution in the space $L_{1}\left(\mathbb{R}^{+}\right) \cap L_{\infty}^{0}\left(\mathbb{R}^{+}\right)$.

Proof. We first consider the Wiener-Hopf integral equation,

$$
S(x)=\int_{0}^{\infty} K_{0}(x-t) S(t) d t, \quad x>0,
$$

for a real measurable function $S(x)$, with a kernel $K_{0}$ obeying the assumptions of the theorem.

As it is known (see [20]), equation (15) has a positive bounded solution with the following properties,

$$
\begin{gathered}
S(x) \geq \eta\left(1-\gamma_{+}\right), \quad S(x) \uparrow \text { w.r.t. } \quad x \quad \text { on } \quad \mathbb{R}^{+} \\
\lim _{x \rightarrow \infty} S(x)=\eta, \\
\gamma_{+} \equiv \int_{0}^{\infty} v_{+}(x) d x \in(0,1) .
\end{gathered}
$$

Here the functions $v_{ \pm}(x) \geq 0, v_{ \pm}(x) \in L_{1}\left(\mathbb{R}^{+}\right)$are determined by Engibaryan's nonlinear factorization equations,

$$
v_{ \pm}(x)=K_{0}( \pm x)+\int_{0}^{\infty} v_{\mp}(t) v_{ \pm}(x+t) d t, \quad x>0,
$$

and

$$
\gamma_{-} \equiv \int_{0}^{\infty} v_{-}(x) d x=1, \quad \gamma_{+} \in(0,1)
$$

In the recent work of one of the authors [21], as an auxiliary statement, the following additional properties of the function $S(x)$

$$
\eta-S(x) \in L_{1}\left(\mathbb{R}^{+}\right) \cap L_{\infty}^{0}\left(\mathbb{R}^{+}\right),
$$




$$
\eta-S(x) \geq \eta \int_{x}^{\infty} K_{0}(\tau) d \tau, \quad x \in \mathbb{R}^{+}
$$

were proven. In what follows, we shall make use of inclusion (21) and inequality (22). We introduce the following successive approximations,

$$
\begin{gathered}
f_{0}(x)=\eta-S(x), \\
f_{n+1}=\int_{0}^{\infty} K_{0}(x-t) N_{0}\left(t, f_{n}(t)\right) d t+\int_{0}^{\infty} K_{1}(x+t) N_{1}\left(t, f_{n}(t)\right) d t, \\
n=0,1,2, \ldots, \quad x \in \mathbb{R}^{+} .
\end{gathered}
$$

By induction on $n$, let us prove the following properties of the sequence $\left\{f_{n}(x)\right\}_{n=0}^{\infty}$,

$$
\text { a) } f_{n}(x) \downarrow \text { w.r.t. } \quad n, \quad \text { b) } f_{n}(x) \geq \Phi_{\eta_{0}}(x), \quad n=0,1,2, \ldots
$$

We note that it follows immediately from $(22)$ and $\eta_{0} \in(0, \eta)$ that

$$
\eta \geq f_{0}(x) \geq \eta \int_{x}^{\infty} K_{0}(\tau) d \tau \geq \eta_{0} \int_{x}^{\infty} K_{1}(\tau) d \tau=\Phi_{\eta_{0}}(x) .
$$

By (26) and the properties of the functions $N_{0}$ and $N_{1}$, in 24 we get

$$
\begin{aligned}
& f_{1}(x)=\int_{0}^{\infty} K_{0}(x-t) N_{0}(t, \eta-S(t)) d t+\int_{0}^{\infty} K_{1}(x+t) N_{1}(t, \eta-S(t)) d t \leqslant \\
& \quad \leqslant \int_{0}^{\infty} K_{0}(x-t)(\eta-S(t)) d t+\int_{0}^{\infty} K_{1}(x+t) N_{1}(t, \eta) d t \leqslant \\
& \leqslant \eta \int_{-\infty}^{x} K_{0}(\tau) d \tau-\int_{0}^{\infty} K_{0}(x-t) S(t) d t+\eta \int_{x}^{\infty} K_{1}(\tau) d \tau \leqslant \eta-S(x)=f_{0}(x), \\
& f_{1}(x) \geq \int_{0}^{\infty} K_{1}(x+t) N_{1}\left(t, f_{0}(t)\right) d t \geq \int_{0}^{\infty} K_{1}(x+t) N_{1}\left(t, \Phi_{\eta_{0}}(t)\right) d t \geq \\
& \geq \eta_{0}^{\infty} \int_{x}^{\infty} K_{1}(\tau) d \tau=\Phi_{\eta_{0}}(x) .
\end{aligned}
$$

Suppose now that $\Phi_{\eta_{0}}(x) \leqslant f_{n}(x) \leqslant f_{n-1}(x)$ for some $n \in \mathbb{N}, x \in \mathbb{R}^{+}$. Then by (24), (14), and the monotonicity of $N_{0}$ and $N_{1}$ we have

$$
\begin{gathered}
f_{n+1}(x) \leqslant \int_{0}^{\infty} K_{0}(x-t) N_{0}\left(t, f_{n-1}(t)\right) d t+\int_{0}^{\infty} K_{1}(x+t) N_{1}\left(t, f_{n-1}(t)\right) d t=f_{n}(x), \\
f_{n+1}(x) \geq \int_{0}^{\infty} K_{1}(x+t) N_{1}\left(t, \Phi_{\eta_{0}}(t)\right) d t \geq \Phi_{\eta_{0}}(x) .
\end{gathered}
$$

Therefore, the sequence of the functions $\left\{f_{n}(x)\right\}_{n=0}^{\infty}$ has a pointwise limit as $n \rightarrow \infty$, $\lim _{n \rightarrow \infty} f_{n}(x)=f(x)$.

By condition (11) and the Lebesgue's dominated convergence theorem (see [22]) it follows that $f(x)$ satisfies equation (1). Moreover, properties (25) imply the following inequalities for the limiting function $f(x)$,

$$
\Phi_{\eta_{0}}(x) \leqslant f(x) \leqslant \eta-S(x) .
$$


Since $\eta-S(x) \in L_{1}\left(\mathbb{R}^{+}\right) \cap L_{\infty}^{0}\left(\mathbb{R}^{+}\right)$, by 27$)$ we obtain that $f(x)>0, f \in L_{1}\left(\mathbb{R}^{+}\right) \cap L_{\infty}^{0}\left(\mathbb{R}^{+}\right)$. The proof is complete.

\section{Solvability of EQUATion (1) FOR EVEn KeRnel $K_{0}$}

We proceed to solving equation (1) under other assumptions for the functions $N_{0}$ and $N_{1}$ in the case

$$
K_{0}(-x)=K_{0}(x), \quad x \in \mathbb{R}^{+} .
$$

The following theorem holds true.

Theorem 2. Given a measurable function $Q: \mathbb{R} \rightarrow \mathbb{R}$, let $\zeta$ and $\eta$ be the lowest positive roots of the equations $Q(x)=2 x$ and $Q(x)=x$, respectively, and $2 \zeta<\eta, Q \in C[0, \eta], Q(x) \uparrow$ w.r.t. $x$ on $[0, \eta]$. Suppose that

a) $0 \leqslant N_{0}(t, z) \leqslant \eta-Q(\eta-z)$ as $(t, z) \in \mathbb{R}^{+} \times[0, \eta]$,

b) $N_{0}, N_{1} \in \operatorname{Carat}_{z}\left(\mathbb{R}^{+} \times[0, \eta]\right)$,

c) $N_{0}, N_{1} \uparrow$ w.r.t. $z$ on the segment $[0, \eta]$ for each fixed $t \in \mathbb{R}^{+}$,

d) there exists $\eta_{0} \in(0, \eta)$ such that

$$
N_{1}\left(t, \Phi_{\eta_{0}}(t)\right) \geq \eta_{0}, \quad N_{1}(t, \eta) \geq \eta .
$$

Then under conditions (4), (5), (28), equation (1) has a positive solution in the space $L_{\infty}^{0}\left(\mathbb{R}^{+}\right)$.

Proof. We consider first the following auxiliary nonlinear Hammerstein type integral equation

$$
\psi(x)=\int_{0}^{\infty} K_{0}(x-t) Q(\psi(t)) d t, \quad x \in \mathbb{R}^{+}
$$

w.r.t. the function $\psi(x)$. We define the iterations,

$$
\psi_{n+1}(x)=\int_{0}^{\infty} K_{0}(x-t) Q\left(\psi_{n}(t)\right) d t, \quad \psi_{0}(x) \equiv \eta, \quad n=0,1,2, \ldots
$$

Due to the properties of the functions $Q$ and $K_{0}$, by the induction on $n$, one can easily make sure that

$$
\psi_{n}(x) \quad \downarrow \text { w.r.t. } \quad n, \quad \psi_{n}(x) \geq \zeta, \quad n=0,1,2, \ldots, \quad x \in \mathbb{R}^{+} .
$$

Therefore, the sequence of the functions $\left\{\psi_{n}(x)\right\}_{n=0}^{\infty}$ has a pointwise limit $\lim _{n \rightarrow \infty} \psi_{n}(x)=\psi(x)$ and by the Levi's theorem the limiting function satisfies equation (29) and the relation

$$
\zeta \leqslant \psi(x) \leqslant \eta, \quad x \in \mathbb{R}^{+} .
$$

By the induction it is also possible to prove that

$$
\psi_{n}(x) \quad \uparrow \text { w.r.t. } \quad x \quad \text { on } \quad \mathbb{R}^{+}, \quad n=0,1,2, \ldots
$$

if one write iterations (30) as follows,

$$
\psi_{n+1}(x)=\int_{-\infty}^{x} K_{0}(\tau) Q\left(\psi_{n}(x-\tau)\right) d \tau, \quad \psi_{0}(x) \equiv \eta, \quad n=0,1,2, \ldots
$$

Hence, in view of $(32)$, we obtain that

$$
\psi(x) \quad \uparrow \text { w.r.t. } \quad x \quad \text { on } \quad \mathbb{R}^{+} .
$$

Thus, due to (31) and (34) we can say that there exists

$$
\lim _{x \rightarrow \infty} \psi(x) \equiv \eta^{*} \leqslant \eta, \quad \eta^{*}>0 .
$$


Passing in 29 to the limit as $x \rightarrow \infty$, by employing the known property of the convolutions and formula (4), we get $\eta^{*}=Q\left(\eta^{*}\right)$. Since $\eta$ is a first positive root of the equation $Q(x)=x$ and $0<\eta^{*} \leqslant \eta$, we have $\eta^{*}=\eta$.

Therefore,

$$
0 \leqslant \eta-\psi \in L_{\infty}^{0}\left(\mathbb{R}^{+}\right)
$$

Let us prove the following auxiliary inequality,

$$
\eta-\psi(x) \geq \eta \int_{x}^{\infty} K_{0}(\tau) d \tau, \quad x \in \mathbb{R}^{+} .
$$

By (29), (4), and the properties of the function $Q$ we have

$$
\begin{gathered}
\eta-\psi(x)=\eta-\int_{0}^{\infty} K_{0}(x-t) Q(\psi(t)) d t=\eta \int_{x}^{\infty} K_{0}(\tau) d \tau+\eta \int_{-\infty}^{x} K_{0}(\tau) d \tau- \\
-\int_{0}^{\infty} K_{0}(x-t) Q(\psi(t)) d t=\eta \int_{x}^{\infty} K_{0}(\tau) d \tau+\int_{0}^{\infty} K_{0}(x-t)(Q(\eta)-Q(\psi(t))) d t \geq \eta \int_{x}^{\infty} K_{0}(t) d t .
\end{gathered}
$$

Consider the following iterations for equation (1),

$$
\left\{\begin{array}{l}
f_{n+1}(x)=\int_{0}^{\infty} K_{0}(x-t) N_{0}\left(t, f_{n}(t)\right) d t+\int_{0}^{\infty} K_{1}(x+t) N_{1}\left(t, f_{n}(t)\right) d t \\
f_{0}(x)=\Phi_{\eta_{0}}(x), \quad n=0,1,2, \ldots \quad x \in \mathbb{R}^{+} .
\end{array}\right.
$$

By induction, we first prove that

$$
f_{n}(x) \quad \uparrow \text { w.r.t. } \quad n \text {. }
$$

Since

$$
0 \leqslant f_{0}(x) \leqslant \eta \int_{x}^{\infty} K_{1}(z) d z \leqslant \eta \int_{x}^{\infty} K_{0}(z) d z
$$

then

$$
\begin{aligned}
f_{1}(x) \geq \int_{0}^{\infty} K_{1}(x+t) N_{1}\left(t, f_{0}(t)\right) d t \geq \Phi_{\eta_{0}}(x) \equiv f_{0}(x), \\
f_{1}(x) \leqslant \int_{0}^{\infty} K_{0}(x-t) N_{0}(t, \eta) d t+\int_{0}^{\infty} K_{1}(x+t) N_{1}(t, \eta) d t \leqslant \eta \int_{-\infty}^{x} K_{0}(\tau) d \tau+ \\
+\eta \int_{x}^{\infty} K_{1}(\tau) d \tau \leqslant \eta .
\end{aligned}
$$

Assuming $\eta \geq f_{n}(x) \geq f_{n-1}(x)$ for some $n \in \mathbb{N}$, by (38), conditions c) and d) we get

$$
f_{n+1}(x) \geq \int_{0}^{\infty} K_{0}(x-t) N_{0}\left(t, f_{n-1}(t)\right) d t+\int_{0}^{\infty} K_{1}(x+t) N_{1}\left(t, f_{n-1}(t)\right) d t=f_{n}(x)
$$

and

$$
f_{n+1}(x) \leqslant \int_{0}^{\infty} K_{0}(x-t) N_{0}(t, \eta) d t+\int_{0}^{\infty} K_{1}(x+t) N_{1}(t, \eta) d t \leqslant \eta
$$


Let us make sure that the inequality

$$
f_{n}(x) \leqslant \eta-\psi(x), \quad n=0,1,2, \ldots, \quad x \in \mathbb{R}^{+}
$$

holds true. Indeed, as $n=0$, (37) implies immediately (41). Let $f_{n}(x) \leqslant \eta-\psi(x)$ for some $n \in \mathbb{N}$. Then by (38) and conditions a) and d) we get

$$
\begin{aligned}
f_{n+1}(x) \leqslant & \int_{0}^{\infty} K_{0}(x-t) N_{0}(t, \eta-\psi(t)) d t+\int_{0}^{\infty} K_{1}(x+t) N_{1}(t, \eta-\psi(t)) d t \leqslant \\
\leqslant & \int_{0}^{\infty} K_{0}(x-t)(\eta-Q(\psi(t))) d t+\int_{0}^{\infty} K_{1}(x+t) N_{1}(t, \eta) d t \leqslant \\
& \leqslant \eta \int_{-\infty}^{x} K_{0}(\tau) d \tau-\psi(x)+\eta \int_{x}^{\infty} K_{1}(\tau) d \tau \leqslant \eta-\psi(x) .
\end{aligned}
$$

Therefore, (40) and (41) yield the pointwise convergence of the sequence $\left\{f_{n}(x)\right\}_{n=0}^{\infty}$ : $\lim _{n \rightarrow \infty} f_{n}(x)=f(x)$ and

$$
0 \leqslant \Phi_{\eta_{0}}(x) \leqslant f(x) \leqslant \eta-\psi(x) \in L_{\infty}^{0}\left(\mathbb{R}^{+}\right), \quad x>0 .
$$

By Levi's theorem, $f(x)$ solves equation (1). It follows from 42 that $f \in L_{\infty}^{0}\left(\mathbb{R}^{+}\right)$. The proof is complete.

Remark 1. The results of Theorem 2 remain true if we replace condition (28) by a weaker one, $\int_{-\infty}^{0} K_{0}(\tau) d \tau \geq \frac{1}{2}$.

\section{Examples of FunCtions $N_{0}, N_{1}$, AND $Q$}

In what follows we give several examples of functions $N_{0}, N_{1}$, and $Q$ subject to the assumptions of the proven theorems.

Examples for Theorem 1.

I) $\quad N_{0}(t, z) \equiv h(t, z) \widetilde{N}(z)$, where the function $h$ is continuous w.r.t. all its arguments on the set $\mathbb{R}^{+} \times[0, \eta], 0 \leqslant h(t, z) \leqslant 1,(t, z) \in \mathbb{R}^{+} \times[0, \eta], h \uparrow$ in $z$ on $[0, \eta], \tilde{N} \in C[0, \eta], \tilde{N} \uparrow$ in $z$ on $[0, \eta], 0 \leqslant \widetilde{N}(z) \leqslant z, z \in[0, \eta]$. As the functions $h$ and $\widetilde{N}$, we can take the following examples,

- $h(t, z)=z e^{-z} \cdot \sin ^{2} t, \quad \tilde{N}(z)=z^{p}, \quad p>1, \quad \eta=1$.

- $h(t, z)=\eta e^{\frac{z}{\eta}-1}, \quad \widetilde{N}(z)=\sin z$.

II)

$$
\begin{aligned}
& N_{1}(t, z)=\frac{\alpha z}{z+\left(\frac{\alpha}{\eta_{0}}-1\right) \Phi_{\eta_{0}}(t)}, \quad \eta>\alpha>\eta_{0}>0, \\
& N_{1}(t, z)=\frac{\alpha z}{z+\left(\frac{\alpha}{\eta_{0}}-1\right) \Phi_{\eta_{0}}(t)}+\frac{1}{2 \eta^{p-1}} z^{p}, \quad p>1, \quad \eta \geq 2 \alpha, \quad \alpha>\eta_{0} .
\end{aligned}
$$

\section{Examples for Theorem 2.}

III) $Q(z)=\frac{z^{\alpha}}{\eta^{\alpha-1}}, \quad \alpha \in(0,1)$,

IV) $Q(z)=\eta e^{\frac{z}{\eta}-1}$

V) $Q(z)=\sqrt{z e^{z-1}}, \quad \eta=1$ 
VI) $\quad N_{0}(t, z)=\frac{(\eta-Q(\eta-z))^{\beta}}{\eta^{\beta-1}}, \quad \beta \geq 1$

VII) $N_{0}(t, z)=\sin (\eta-Q(\eta-z))$

As $N_{1}(t, z)$, in Theorem 2 we can consider examples $(43 a)$ and $(43 b)$.

\section{On solvability of problem (2)-(3) in Sobolev Space $W_{1}^{1}\left(\mathbb{R}^{+}\right)$}

The following theorem holds true.

Theorem 3. Suppose the function $H(t, z)$ in equation (2) satisfies all the assumptions for the function $N_{0}(t, z)$ in Theorem 1 , and $H_{1}(t, z)$ is a real function defined on the set $\mathbb{R}^{+} \times \mathbb{R}$ and there exist positive numbers $\eta>0, \eta_{0} \in(0, \eta), \xi \in\left(0, \frac{1}{\lambda}\right), \theta \in(0,1)$ such that

$$
\left.i_{1}\right) \quad H_{1}\left(t, \xi \rho_{\eta_{0}}^{\sigma}(t)\right) \geq \eta_{0}, \quad H_{1}(t, \eta) \leqslant \eta
$$

where

$$
\begin{array}{r}
\rho_{\eta_{0}}^{\sigma}(t)=\eta_{0} \int_{t+\sigma}^{\infty} T_{1}(z) d z, \quad \sigma=\frac{1}{\lambda \theta} \ln \frac{1}{1-\lambda \xi} \\
\left.i_{2}\right) \quad H_{1}(t, 0) \equiv 0, \quad H_{1} \in \operatorname{Carat}_{z}\left(\mathbb{R}^{+} \times[0, \eta]\right) .
\end{array}
$$

$\left.i_{3}\right) \quad H_{1}(t, z) \uparrow$ w.r.t. $z$ on $[0, \eta]$ for each fixed $t \in \mathbb{R}^{+}$.

Then under conditions (6)-(9), problem (2)-(3) has a nonnegative nontrivial solution in the Sobolev space $W_{1}^{1}\left(\mathbb{R}^{+}\right)$.

Proof. We introduce the function

$$
K_{0}(x)=\int_{0}^{\infty} e^{-\lambda z} T(x-z) d z, \quad x \in \mathbb{R} .
$$

By the Fubini theorem, the function $K_{0}(x)$ possesses the following "splendid" properties,

$$
\begin{gathered}
K_{0}(x) \geq 0, \quad \int_{-\infty}^{+\infty} K_{0}(x) d x=1, \quad K_{0} \in L_{1}(\mathbb{R}) \cap L_{\infty}(\mathbb{R}), \\
\nu\left(K_{0}\right)<0, \quad \int_{-\infty}^{+\infty} \tau^{2} K_{0}(\tau) d \tau<+\infty
\end{gathered}
$$

Let us prove the following inequality

$$
\int_{x}^{\infty} K_{0}(t) d t \geq \frac{1}{\lambda} \int_{x}^{\infty} T(t) d t, \quad x \in \mathbb{R}^{+} .
$$

We have

$$
\begin{aligned}
\int_{x}^{\infty} K_{0}(t) d t & =\int_{x}^{\infty} \int_{0}^{\infty} e^{-\lambda z} T(t-z) d z d t=\int_{0}^{\infty} e^{-\lambda z} \int_{x}^{\infty} T(t-z) d t d z= \\
& =\int_{0}^{\infty} e^{-\lambda z} \int_{x-z}^{\infty} T(y) d y d z \geq \frac{1}{\lambda} \int_{x}^{\infty} T(t) d t .
\end{aligned}
$$

Consider the homogeneous Wiener-Hopf equation

$$
S(x)=\int_{0}^{\infty} K_{0}(x-t) S(t) d t, \quad x \in \mathbb{R}^{+},
$$


with a kernel of the form (47). As it was noted, (48), (49) imply the existence of a positive solution with properties (16), (17), (21), (22).

Denote

$$
F(x)=\frac{d \varphi}{d x}+\lambda \varphi(x) .
$$

Then equation (2) with initial condition (3) casts into the form

$$
\begin{gathered}
F(x)=\int_{0}^{\infty} T(x-t) H\left(t, \int_{0}^{t} e^{-\lambda(t-\tau)} F(\tau) d \tau\right) d t+ \\
+\int_{0}^{\infty} T_{1}(x+t) H_{1}\left(t, \int_{0}^{t} e^{-\lambda(t-\tau)} F(\tau) d \tau\right) d t, \quad x \in \mathbb{R}^{+} .
\end{gathered}
$$

Consider the iterations

$$
\begin{aligned}
& F_{n+1}(x)=\int_{0}^{\infty} T(x-t) H\left(t, \int_{0}^{t} e^{-\lambda(t-\tau)} F_{n}(\tau) d \tau\right) d t+ \\
& +\int_{0}^{\infty} T_{1}(x+t) H_{1}\left(t, \int_{0}^{t} e^{-\lambda(t-\tau)} F_{n}(\tau) d \tau\right) d t \\
& F_{0}(x)=\lambda(\eta-S(x)), \quad n=0,1,2, \ldots, \quad x \in \mathbb{R}^{+} .
\end{aligned}
$$

In what follows we shall show that

$$
\begin{array}{ll}
\left.j_{1}\right) \quad F_{n}(x) \downarrow \text { w.r.t. } n, \\
\left.j_{2}\right) \quad F_{n}(x) \geq \rho_{\eta_{0}}^{\sigma}(x), \quad n=0,1,2, \ldots, \quad x \in \mathbb{R}^{+} .
\end{array}
$$

By 22 and 50 we have

$$
\begin{aligned}
F_{0}(x)=\lambda(\eta-S(x)) & \geq \lambda \eta \int_{x}^{\infty} K_{0}(t) d t \geq \eta \int_{x}^{\infty} T(t) d t \geq \eta \int_{x}^{\infty} T_{1}(t) d t \geq \\
& \geq \eta_{0} \int_{x+\sigma}^{\infty} T_{1}(t) d t=\rho_{\eta_{0}}^{\sigma}(x) .
\end{aligned}
$$

In particular, it implies that

$$
\rho_{\eta_{0}}^{\sigma}(x) \leqslant \lambda \eta, \quad x \in \mathbb{R}^{+} .
$$

Employing the properties of the functions $H, H_{1}, T$, and $T_{1}$, we obtain

$$
\begin{gathered}
F_{1}(x) \leqslant \int_{0}^{\infty} T(x-t) H\left(t, \eta-\lambda \int_{0}^{t} e^{-\lambda(t-\tau)} S(\tau) d \tau\right) d t+\int_{0}^{\infty} T_{1}(x+t) H_{1}(t, \eta) d t \leqslant \\
\leqslant \eta \int_{0}^{\infty} T(x-t) d t-\lambda \int_{0}^{\infty} T(x-t) \int_{0}^{t} e^{-\lambda(t-\tau)} S(\tau) d \tau d t+\eta \int_{x}^{\infty} T_{1}(z) d z \leqslant \\
\leqslant \lambda \eta-\lambda \int_{0}^{\infty} K_{0}(x-\tau) S(\tau) d \tau=\lambda(\eta-S(x))=F_{0}(x) .
\end{gathered}
$$


Let $F_{n}(x) \geq \rho_{\eta_{0}}^{\sigma}(x)$ for some $n \in \mathbb{N}$. Then by (44), (45), (54), $\left.i_{3}\right)$, monotonicity of $H(t, z)$ we obtain

$$
\begin{aligned}
F_{n+1}(x) \geq \int_{0}^{\infty} T(x-t) H\left(t, \int_{0}^{t} e^{-\lambda(t-\tau)} \rho_{\eta_{0}}^{\sigma}(\tau) d \tau\right) d t+ \\
\quad+\int_{0}^{\infty} T_{1}(x+t) H_{1}\left(t, \int_{0}^{t} e^{-\lambda(t-\tau)} \rho_{\eta_{0}}^{\sigma}(\tau) d \tau\right) d t \geq \\
\quad \geq \int_{0}^{\infty} T_{1}(x+t) H_{1}\left(t, \int_{0}^{t} e^{-\lambda(t-\tau)} \rho_{\eta_{0}}^{\sigma}(\tau) d \tau\right) d t \geq \\
\geq \int_{\sigma}^{\infty} T_{1}(x+t) H_{1}\left(\begin{array}{c}
t, \int_{(1-\theta) \sigma}^{t} e^{-\lambda(t-\tau)} \rho_{\eta_{0}}^{\sigma}(\tau) d \tau \\
t
\end{array}\right) d t \geq \\
\geq \int_{\sigma}^{\infty} T_{1}(x+t) H_{1}\left(t, \rho_{\eta_{0}}^{\sigma}(t) \int_{(1-\theta) \sigma}^{\sigma} e^{-\lambda(\sigma-\tau)} d \tau\right) d t \geq \\
\geq \int_{\sigma}^{\infty} T_{1}(x+t) H_{1}\left(t, \rho_{\eta_{0}}^{\sigma}(t) \frac{\left(1-e^{-\lambda \theta \sigma}\right)}{\lambda}\right) d t= \\
=\int_{\sigma}^{\infty} T_{1}(x+t) H_{1}\left(t, \xi \rho_{\eta_{0}}^{\sigma}(t)\right) d t \geq \eta^{\infty} T_{1}^{\infty}(y) d y=\rho_{\eta_{0}}^{\sigma}(x) .
\end{aligned}
$$

Suppose $F_{n}(x) \leqslant F_{n-1}(x)$ for some $n \in \mathbb{N}$. Then the monotonicity of $H$ and $H_{1}$ immediately yields that $F_{n+1} \leqslant F_{n}$. Therefore, there exists

$$
\lim _{n \rightarrow \infty} F_{n}(x)=F(x)
$$

and $F(x)$ satisfies equation (53) and the estimates

$$
\rho_{\eta_{0}}^{\sigma}(x) \leqslant F(x) \leqslant \lambda(\eta-S(x)) \in L_{1}\left(\mathbb{R}^{+}\right) \cap L_{\infty}^{0}\left(\mathbb{R}^{+}\right) .
$$

It follows from (59) that $F \in L_{1}\left(\mathbb{R}^{+}\right) \cap L_{\infty}^{0}\left(\mathbb{R}^{+}\right)$.

Solving the simplest Cauchy problem

$$
\left\{\begin{array}{l}
\frac{d \varphi}{d x}+\lambda \varphi(x)=F(x), \quad x \in \mathbb{R}^{+} \\
\varphi(0)=0
\end{array}\right.
$$

we complete the proof.

Remark 2. Since a solution to problem reads as

$$
\varphi(x)=\int_{0}^{x} e^{-\lambda(x-t)} F(t) d t
$$

by (59) we get the following two-sided estimate

$$
\int_{0}^{x} e^{-\lambda(x-t)} \rho_{\eta_{0}}^{\sigma}(t) d t \leqslant \varphi(x) \leqslant \lambda \int_{0}^{x} e^{-\lambda(x-t)}(\eta-S(t)) d t
$$

for $\varphi(x)$. 
In the end of the work, we give two examples of $H_{1}(t, z)$,

$$
\begin{aligned}
& \text { 1) } H_{1}(t, z)=\frac{\alpha z}{z+\left(\frac{\alpha}{\eta_{0}}-1\right) \rho_{\eta_{0}}^{\sigma}(t)}, \quad \eta>\alpha>\eta_{0}>0, \\
& \text { 2) } H_{1}(t, z)=\frac{\alpha z}{z+\left(\frac{\alpha}{\eta_{0}}-1\right) \rho_{\eta_{0}}^{\sigma}(t)}+\frac{1}{2 \eta^{p-1}} z^{p}, \quad p>1, \quad \eta \geq 2 \alpha, \quad \alpha>\eta_{0} .
\end{aligned}
$$

In conclusion we express out gratitude to prof. N.B. Engibaryan and prof. V.N. Margaryan for useful advices.

\section{BIBLIOGRAPHY}

1. M.N. Kogan. Rarefied gas dynamics. Nauka, Moscow. 1962. [Plenum, New York. 1969.]

2. N.B. Engibaryan, A.Kh. Khachatryan. Exact linearization of the sliding problem for a dilute gas in the Bhatnagar-Gross-Krook model // eor. Matem. Fiz. 2000. V. 119, No. 2. P. 339-342. [Theor. Math. Phys. 2000. V. 119, No. 2. P. 1589-1592.]

3. J.D. Sargan. The distribution of wealth // Econometrics. 1957. V. 25, No. 4. P. 568-590.

4. A.Kh. Khachatryan, Kh.A. Khachatryan. On integro-differential equation in Sana problem of wealth distribution // Ekonomika i matem. metody, CEMI RAS. 2009. V. 45, No. 4. P. 84-96. (in Russian.)

5. F.D. Gakhov. Yu.I. Cherskii. Convolution type equations. Nauka, Moscow. 1978.

6. B.N. Engibaryan. Application of multiple factorization to convolution-type homogeneous equation // Izv. NAN Armenii, Matem. 1997. V. 32, No. 1. P. 38-48. (in Russian).

7. N.B. Engibaryan, A.Kh. Khachatryan. Some convolution-type integral equations in kinetic theory // Zhur. Vychisl. Matem. Matem. Fiz. 1998. V. 38, No. 3. P. 466-482. [Comp. Math. Math. Phys. 1998. V. 38, No. 3, P. 452-467.]

8. N.B. Engibaryan, L.G. Arabadzhyan. Some factorization problems for convolution integral operators // Diff. Uravn. 1990. V. 26, No. 1. P. 1442-1452. [Diff. Equat. 1990. V. 26, No. 1. P. 1069-1078.]

9. V.S. Vladimirov. The equation of the p-adic open string for the scalar tachyon field // Izv. RAN. Ser. matem. 2005. V. 69, No. 3. P. 55-80. [Izv. Math. 2005. V. 69, No. 3. P. 487-512.]

10. V.S. Vladimirov, Y.I. Volovich. Nonlinear dynamics equation in p-adic string theory // Teor. Matem. Fiz. 2004. V. 138, No. 3. P. 355-368. [Theor. Math. Phys. 2004. V. 138, No. 3. P. 297309.]

11. P.H. Framton, Y. Okada. Effective scalar field theory of p-adic string // Phys. Rev. D. 2004. V. 37, No. 10. P. 3077-3079.

12. V.S. Vladimirov. The equation of p-adic closed string for the scalar tachyon field // Science in China, ser.A, Mathematics. 2008. V. 51, No. 4. P. 754-764.

13. A.Kh. Khachatryan, Kh.A. Khachatryan. On convolution type nonlinear integral equations, containing singular and discrete probability distributions // Advances and Applications in Mathematical Sciences. India. 2010. V. 5, No. 1, P. 1-16.

14. L.G. Arabadzhyan. Solutions to an integral Hammerstein type equation // Izv. NAN Armenii, Matem. 1997. V. 32, No. 1. P. 21-28.

15. A.Kh. Khachatryan, Kh.A. Khachatryan. On solvability of one class Hammerstein nonlinear integral equations // Buletinul Academiei Stinte a Republici Moldova, Mathematica. 2010. V. 63, No. 2. P. 67-83.

16. Kh.A. Khachatryan. Existence and asymptotic behavior of solution to a class of nonlinear integral Urysohn equation on the half-line // Vestnik RAU. 2009. V. 3, No. 2. P. 15-25.

17. Kh.A. Khachatryan. On a class of nonlinear integral equations with noncompact operator // Izvestia NAN Armenii, Matem. 2011. V. 46, No. 2. P. 71-86.

18. Kh.A. Khachatryan. On a class of integral equations of Urysohn type with strong non-linearity // Izv. RAN. Ser. matem. 2012. V. 76, No. 1. P. 173-200. [Izv. math. 2012. V. 76, No. 1. P. 163-189.]

19. Kh.A. Khachatryan, E.A. Khachatryan. Solvability of some classes of nonlinear integrodifferential equations with noncompact operator // Izv. VUZov. Matem. 2011. V. 54, No. 1. P. 91-100. [Russ. Math. (Izv. VUZ. Matem.) 2011. V. 55, No. 1. P. 79-87.] 
20. L.G. Arabadzhyan, N.B. Engibaryan. Convolution equations and nonlinear functional equations // Itogi nauki i tekhniki. Matem. analiz. 1984. V. 22. P. 175-242. [J. Soviet Math. 1987. V. 36, No. 6. P. 745-791.]

21. A.Kh. Khachatryan, Kh.A. Khachatryan. On Solvability of a nonlinear problem in theoty of income distribution // Eurasian Math. J. 2011. V. 2, No. 2. P. 75-88.

22. A.N. Kolmogorov, V.S. Fomin. Elements of the theory of functions and functional analysis. Nauka, Moscow. 1981. [Dover Publication, New York. 1999.]

Marine Firdousovna Broyan,

Armenian National Agrarian University,

Teryan str., 74,

0019, Erevan, Armenia

E-mail: Broyan@rambler.ru

Khachatur Agavardovich Khachatryan, Institute of Mathematics of NAS RA,

Marshal Baghramian avenue, 24/5,

0019, Erevan, Armenia

E-mail: Khach820rambler.ru 